\title{
INDIVIDUAL TREE SEGMENTATION OVER LARGE AREAS USING AIRBORNE LIDAR POINT CLOUD AND VERY HIGH RESOLUTION OPTICAL IMAGERY
}

\author{
Yuchu Qin ${ }^{1}$, Antonio Ferraz ${ }^{1,2}$, Clément Mallet ${ }^{1}$, Corina Iovan ${ }^{3}$ \\ 1. IGN, Laboratoire MATIS, Université Paris Est, France \\ 2. Instituto de Engenharia de Sistemas e Computadores de Coimbra, Coimbra, Portugal \\ 3. UR-CoRéUs-LabEx-CORAIL, Institut de Recherche pour le Développement, France
}

\begin{abstract}
Timely and accurate measurements of forest parameters are critical for ecosystem studies, sustainable forest resources management, monitoring and planning. This paper presents a processing chain for individual tree segmentation over large areas with airborne LiDAR 3D point cloud and very high resolution (VHR) optical imagery. The proposed processing chain consists of forest stand level delineation with optical imagery, individual tree segmentation with Canopy Height Model (CHM) derived from LiDAR point cloud, rough characterization of trees at forest stand level, and point clustering of individual tree with an Adaptive Mean Shift 3D (AMS3D) algorithm. The processing chain is developed with the expectation of supporting operational forest inventory at individual tree level. Experiment is conducted using LiDAR data acquired in Ventoux region, France. Results suggest that the proposed processing chain can be successfully adopted for individual tree characterization over large areas with different forest stands. Index Terms-Point cloud, VHR imagery, Individual tree segmentation, Forest parameter estimate, Tree level, Large areas.
\end{abstract}

\section{INTRODUCTION}

Forests are the most important terrestrial ecosystems on the Earth's land surface as providing vital goods and fundamental services upon which the welfare of humanity depends. Timely and accurate measurements of forest parameters are critical for understanding the ecological role of forests in the terrestrial ecosystem as well as sustainable forest resources management and carbon accounting [Rosenqvist et al., 2003].

Over the past decade, it has been well established that airborne Light Detection and Ranging (LiDAR) data and high resolution remotely sensed imagery provide the capability of acquiring detailed forest structural parameters [Asner et al., 2010]. However, for large area characterization of vegetation structure using remotely sensed data, the spatial extent and spatial resolution of a given sensor are inversely related [Wulder et al., 2012]. Hence, estimation of detailed forest parameters such as diameter at breast height (DBH), stem volume, and basal area was limited to small areas which are not sufficient for operational monitoring. With the increasingly available airborne LiDAR point cloud and VHR optical imagery, operational forest monitoring over large areas at fine resolution has attracted more and more attention [Wulder et al., 2012; Hopkinson et al., 2013].

It has been widely addressed that there are two types of methods for LiDAR based forest inventories: area-based methods and single-tree methods [Hyyppä et al., 2012]. Most existing methods require ground truth data to construct predictive models. However, the collection of field measurements is a time consuming and costly task. To provide an automated approach for estimating tree parameters without field data, we have developed the AMS3D clustering algorithm to model 3D forest structure at individual tree level using point cloud data [Ferraz et al., 2012]. The AMS3D algorithm is tuned by a parameter named bandwidth which depends on the forest stand characteristics (tree height, crown diameter). Due to the forest heterogeneity across the landscape, the AMS3D algorithm cannot handle large-area forest mapping automatically. With the fusion of VHR optical imagery and LiDAR point cloud, the objective of the approach proposed in this paper is to bridge this gap by developing an automated processing framework for estimating forest parameters at individual tree level over large areas.

\section{METHODS}

The proposed processing chain is designed to characterize forest structure at individual tree level over large areas. It is based on the AMS3D algorithm which is directly applied on LiDAR 3D point cloud to model individual trees within forest patches, using the optimal bandwidth determined by the VHR imagery segmentation at forest stand level. Figure 1 illustrates the technical workflow of the proposed processing framework. The workflow consists of five main steps:1). Generation of Digital Elevation Model (DEM) and CHM from LiDAR point cloud; 2). Multi-resolution image segmentation for forest patches delineation at stand level; 3 ). 
Individual tree crown delineation with LiDAR based CHM; 4).Tree characteristics at forest stand level; 5). AMS3D based point cloud clustering.

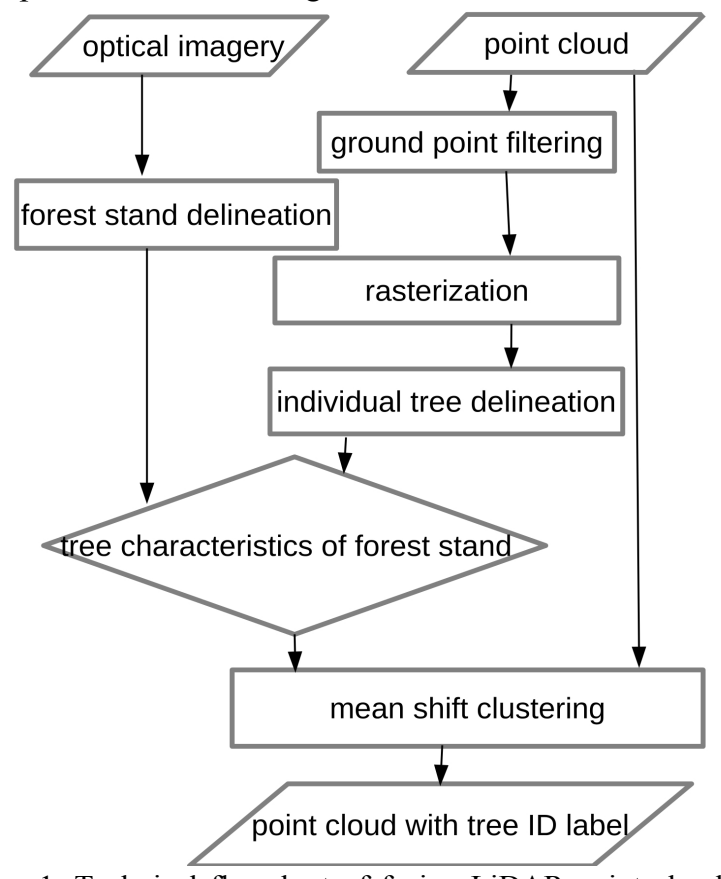

Figure 1. Technical flowchart of fusing LiDAR point cloud and high resolution imagery for individual tree segmentation.

\subsection{Generation of Digital Elevation Model (DEM) and CHM from LiDAR point cloud}

3D LiDAR point cloud data is firstly classified as ground/non-ground points using Terrasolid. DEM and CHM can be generated with the classified LiDAR point cloud data.

Ground points, i.e., points reflected by ground surfaces are extracted to construct Triangulated Irregular Network (TIN). DEM grid is generated by interpolating the adjacent nodes of DEM pixels in TIN.

The non-ground points in the original point cloud data are normalized by substracting the height of corresponding DEM pixels. In this study, we assume the non-ground points with a height greater than $2.0 \mathrm{~m}$ is reflected by tree canopies, so that the points with normalized height greater than $2.0 \mathrm{~m}$ are extracted to generate the CHM.

\subsection{VHR image segmentation for forest stand delineation}

Image segmentation is performed at forest stand level to delineate homogeneous forest patches assuming that trees have similar characteristics (tree density, growth stage and crown size) at such object level. Although high heterogeneity is the nature of forest landscape over large areas, the process of image segmentation is to partition digital image into multiple homogeneous patches featuring similar characteristics. Hence, the forest stand level delineation is a challenging task.

The multi-resolution segmentation (MRS) algorithm was developed by [Benz et al., 2004]. In the past decade, MRS algorithm has been widely applied in remote sensing image segmentation. In this study, the algorithm is applied for high resolution image segmentation, and it is expected to generate homogeneous forest patches to parameterize the AMS3D algorithm.

\subsection{Individual tree crown detection with CHM}

To provide a rough estimation of medium crown size at forest stand level, an approach based on multi-scale gradient transformation and stepwise image segmentation is applied on CHM for Individual Tree Crown (ITC) delineation. A multi-scale slope gradient transformation approach is proposed to highlight the slope over tree canopies with a Gradient Comparison Ratio (GCR). It is expected to provide a normalize variable to describe slope characteristics of individual tree canopies without the consideration of absolute tree height. For a given pixel, GCR of a particular window size is the ratio between the number of pixels at outer border with a lower elevation and the number of overall pixels at outer borders. With the summation of multi-scale GCR, treetops are identified with a flood-fill algorithm, based on the detected tree-top locations; finally, a marker-controlled watershed segmentation is carried out to identify the crown of individual trees.

\subsection{Forest stand characterization}

To characterize the crown size of each forest patch, overlay analysis between forest stand segmentation and individual tree crown delineation is applied to identify all detected trees in each forest stand level segmentation. For a given forest patch, the medium tree crown size is identified by taking into account all trees located in the segment. However, the ITC delineation may have over-segmented and/or under-segmented objects. Hence, the overlay analysis step will only be performed on ITC segments satisfying the following criteria: 1). Area is greater than $5.0 \mathrm{~m}^{2} ; 2$ ). The ratio between width and height (computed using the bounding box of each segment) is greater than 0.5 or less than 2.0.

2.5. AMS3D based point clustering for individual tree segmentation

The original $\mathrm{Z}$ coordinates of point cloud data are normalized with Digital Elevation Model (DEM) by substracting the elevation of corresponding location. Nonground points with height greater than $2.0 \mathrm{~m}$ are selected for individual tree segmentation.

Although the AMS3D algorithm is a robust approach, for a given forest stand, an optimal bandwidth is still desired to adapt the actual tree characterizations. An appropriate 
bandwidth will not only reduce over-segmentation or undersegmentation, it also reduces the search range and therefore improves the computational efficiency of the point clustering algorithm. Therefore, the medium diameter of tree crown size of segmented forest stand level patch is adopted as the optimal bandwidth to parameterize the AMS3D algorithm.

For a given forest stand level patch, the normalized nonground LiDAR points falling in the area are extracted for the individual tree segmentation. With the optimal bandwidth, individual tree segmentation is performed at forest stand level patch by the AMS3D algorithm, and non-ground LiDAR points are assigned to a specific individual tree with a unique label.

\section{EXPERIMENT AND RESULTS}

\subsection{Study area and dataset}

The study area is located in the Ventoux region, South-East France. LiDAR point cloud is acquired with an $\mathrm{OPTECH}$ 3100 system in 2012 , operated with a scan angle of $14^{\circ}$, and with a scanning frequency of $50 \mathrm{kHz}$. Four-channel (NIR, red, green, blue) very high resolution aerial imagery is simultaneously acquired by camV2, which is developed by IGN, France for national mapping applications [Souchon et al., 2012]. Orthoimages are produced using the original aerial images and are tiled as $1000 \mathrm{~m} * 1000 \mathrm{~m}$ standard product with $0.5 \mathrm{~m}$ spatial resolution[Boldo et al., 2000].

\subsection{Results}

Figure 2 shows the true color orthoimage with forest stand level segmentation, it is observed that each segmented forest patch has similar characteristics and keep clear boundaries with neighbor patches.

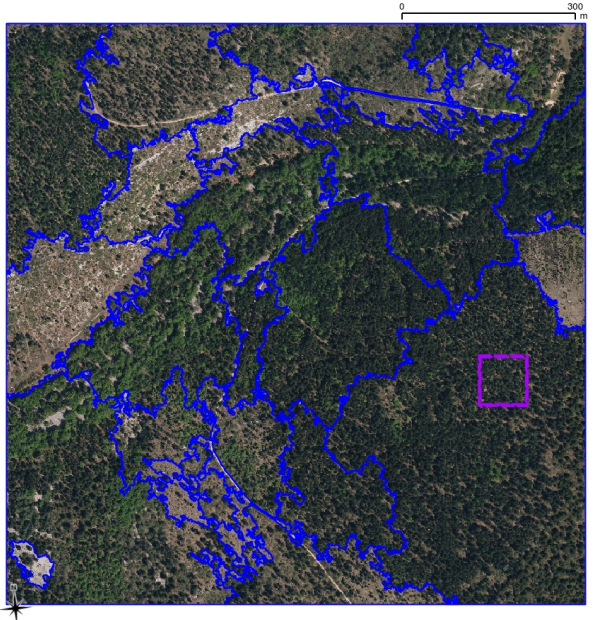

Figure 2. True color orthoimage with forest stand level segmentation (blue).
In Figure 3, CHM based ITC delineation results are illustrated for an area highlighted as purple rectangle in Figure 2. Visual inspection upon the CHM and delineations suggests that ITC could be detected with the multi-scale gradient transformation and stepwise image segmentation based approach. However, the result also suggests that many tree crowns are segmented as separate parts, i.e. oversegmented, and sliver segments are also produced. Hence, the unreliable ITC segments are excluded by criterion described in section 2.4 for estimating the bandwidth at forest stand level.

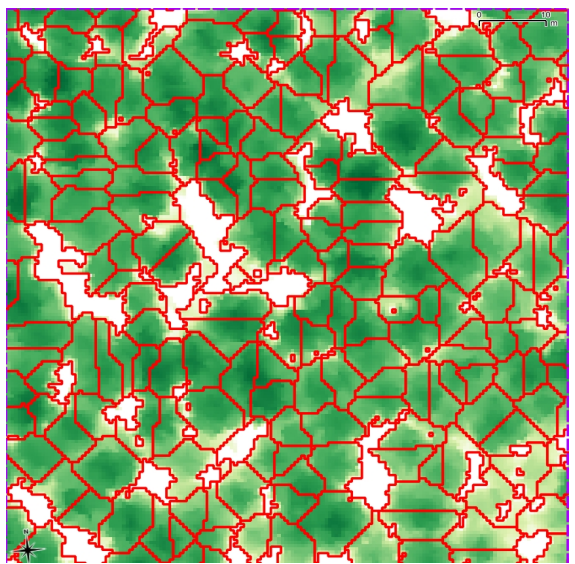

Figure 3. CHM based ITC delineation of a subset area in Figure 2(Rectangle in purple): Red lines are delineation borders, WhiteDark green depicts the height of CHM ranging from $2.0 \mathrm{~m}$ to 20.0 m.

With the stand level bandwidth obtained by overlaying the optical image segmentation and ITC delineation, point clustering is conducted by the AMS3D algorithm to assign each LiDAR point to a specific tree. Figure 4 shows the result of point cloud segmentation of individual trees. Point clusters are assigned different colors to discriminate adjacent trees.

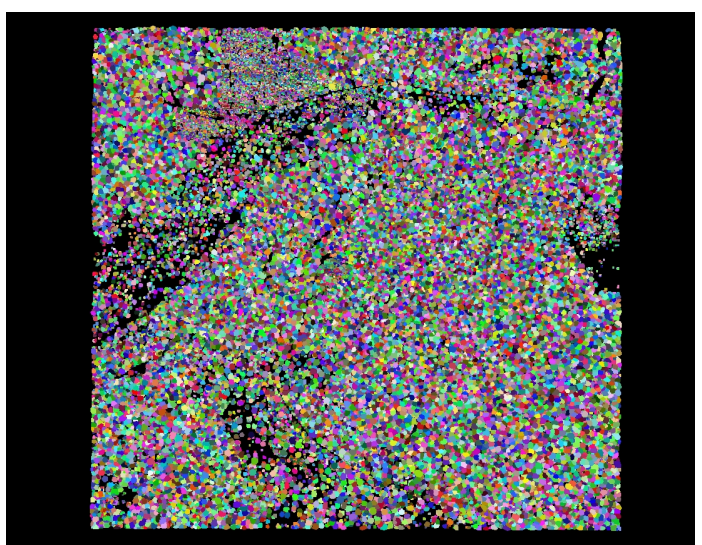

Figure 4. Result of the AMS3D based point clustering (1 color per cluster). 
To provide a detailed scene of the point clustering at individual tree, a forest stand level segment is selected as sample site for 3D visualization. Figure 5. shows labeled point cloud for a segment in Figure 2., It can be observed that points of individual trees are labeled as same clusters.

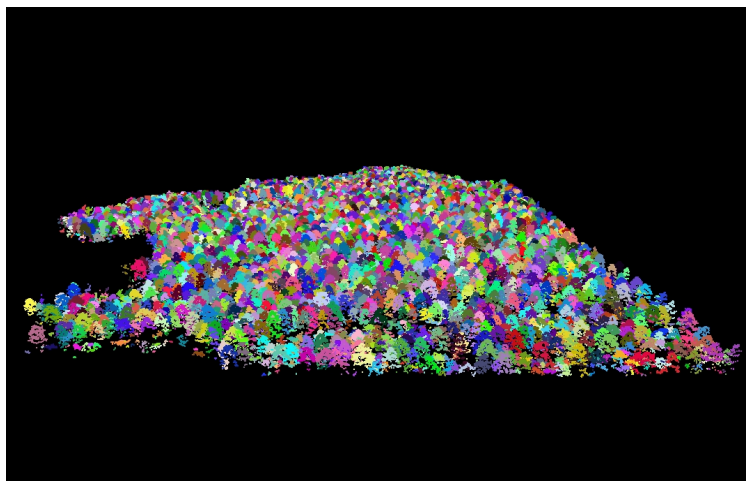

Figure 5. 3D visualization of point cloud with tree labels for the forest stand segment with purple rectangle in Figure 2.

With the individual tree segmentation, forest parameters can be derived. Individual Tree Height (ITH) is estimated to demonstrate the potential applications of the individual tree segmentation results. Figure 6 shows the ITH derived from the individual trees showed in Figure 5. The tree heights are identified using the highest point of tree segments.

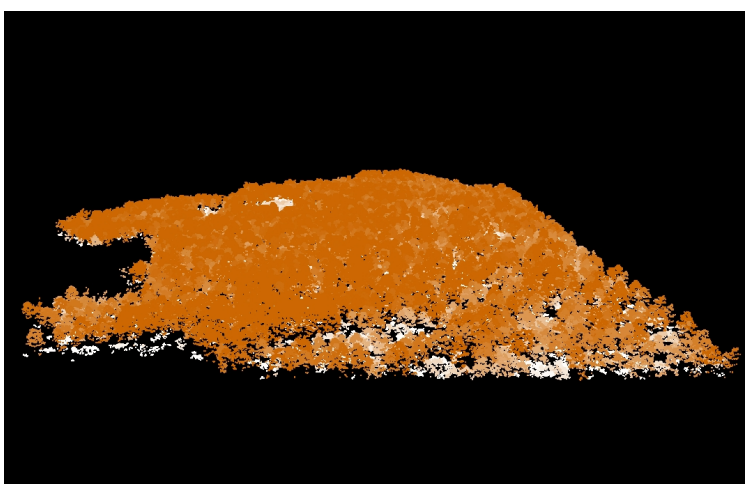

Figure 6. Height of individual trees: White-Yellow depicts tree height ranging from $5.0-25.0 \mathrm{~m}$.

\section{CONCLUSION}

This paper presents an automated processing chain for individual tree segmentation with LiDAR point cloud and high resolution optical imagery over large areas. Results show the proposed approach is a promising solution for large-area forest mapping at individual tree level. Future work will focus on the quantitative assessment of the segmentation as well as deriving high level biophysical forest parameters from the individual tree segmentation over large areas.
Acknowledgements: This work is supported by the French National Research Agency through the FORESEE project (ANR-2010-BIOE-008). The authors would like to thank Laurent Saint-André (INRA) and Jean-PIerre Renaud (ONF) for discussions and providing the allometric equations.

\section{REFERENCES}

[1] Å. Rosenqvist, A. Milne, R. Lucas, M. Imhoff, and C. Dobson, (2003). "A review of remote sensing technology in support of the Kyoto Protocol," Environmental Science \& Policy, pp. 441-455, 2003.

[2] G. P. Asner, G. V. Powell, J. Mascaro, D. E. Knapp, J. K. Clark, and J. Jacobson, et al. "High-resolution forest carbon stocks and emissions in the Amazon," PNAS, pp. 1673816742, 2010.

[3] M. Simard, N. Pinto, J. B. Fisher, and A. Baccini, "Mapping forest canopy height globally with spaceborne lidar," Journal of Geophysical Research: Biogeosciences, 116(G4), 2011.

[4] M. A. Wulder, J. C. White, R. F. Nelson, E. Næsset, H. O. Ørka, and N. C. Coops, "Lidar sampling for large-area forest characterization: A review," Remote Sensing of Environment, pp. 196-209, 2012.

[5] A. Ferraz, F. Bretar, S. Jacquemoud, G. Gonçalves, L. Pereira, M. Tomé, and P. Soares, "3-D mapping of a multilayered Mediterranean forest using ALS data," Remote Sensing of Environment, pp. 210-223, 2012.

[6] C. Hopkinson, L. Chasmer, D. Colville, R. A. Fournier, R. J. Hall, and J. E. Luther, "Moving Toward Consistent ALS Monitoring of Forest Attributes across Canada: A Consortium Approach," Photogrammetric Engineering and Remote Sensing, pp. 159-173, 2013.

[7] J. Hyyppä, X. Yu, H. Hyyppä, M. Vastaranta, M. Holopainen, and A. Kukko, "Advances in forest inventory using airborne laser scanning," Remote Sensing, pp.11901207, 2012.

[8] U. C. Benz, P. Hofmann, G. Willhauck, I. Lingenfelder, and M. Heynen, "Multi-resolution, object-oriented fuzzy analysis of remote sensing data for GIS-ready information," ISPRS Journal of photogrammetry and remote sensing, pp. 239-258, 2004.

[9] J. P. Souchon, C. Thom, C. Meynard, and O. Martin, "A large format camera system for national mapping purposes," Revue Française de Photogrammétrie et de Télédétection, pp. 48-53, 2012.

[10] D. Boldo, H. Le Men. Mosaïque automatique d'orthophotographies. Proceeding of RFIA (Reconnaissance des Formes et Intelligence Artificielle), vol. 3, Paris, France, 2000 . 